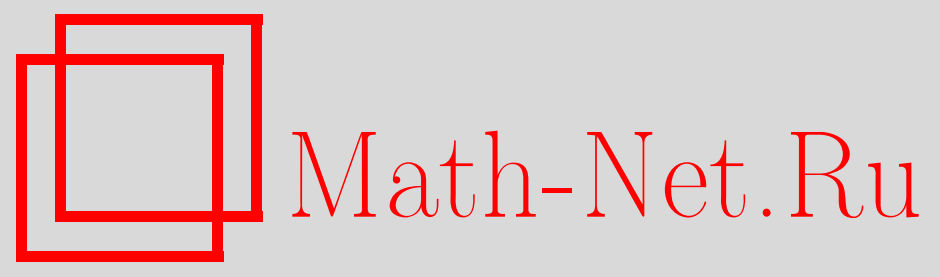

В. Г. Кановей, И. Заплетал, Пирамидальная структура степеней конструктивности, Матем. заметки, 1998, том 63, выпуск 4, 632-635

DOI: https://doi.org/10.4213/mzm1325

Использование Общероссийского математического портала Math-Net.Ru подразумевает, что вы прочитали и согласны с пользовательским соглашением http://www.mathnet.ru/rus/agreement

Параметры загрузки:

IP : 44.207 .124 .84

26 апреля 2023 г., 13:17:54

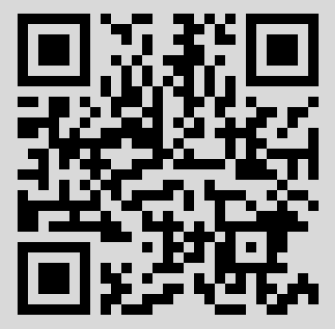




\section{ПИРАМИДАЛЬНАЯ СТРУКТУРА СТЕПЕНЕЙ КОНСТРУКТИВНОСТИ}

\section{В.Г. Кановей, И. Заплетал}

Введение. Рассматривается модель, структура степеней конструктивности которой включает

1) степени $\mathbf{0}<\boldsymbol{a}_{0}<\boldsymbol{a}_{1}<\cdots$, где $\mathbf{0}$ - конструктивная степень;

2) степень $\boldsymbol{b}>\mathbf{0}$, несравнимую ни с одной из $\boldsymbol{a}_{n}$;

3) "конкатенации" $\boldsymbol{a}_{0} \boldsymbol{b}<\boldsymbol{a}_{1} \boldsymbol{b}<\boldsymbol{a}_{2} \boldsymbol{b}<\cdots$;

4) наибольшую степень $\boldsymbol{a} \omega \boldsymbol{b}$.

Степени $\boldsymbol{a}_{0}, \boldsymbol{a}_{1}, \ldots$ и $\boldsymbol{b}$ можно было бы получить при помоши форсинга Sacks ${ }^{\omega} \times$ Sacks. (Здесь Sacks $^{\omega}$ есть итерация форсинга Caкса Sacks длины $\omega$ со счетной “поддержкой", см. [1]. Соответственно Sacks ${ }^{m}$ есть итерация Sacks длины $m$.) Однако, при этом возникает еще одна степень $\boldsymbol{a}_{\omega}-$ верхняя грань степеней $\boldsymbol{a}_{n}, n \in \omega$, которая несравнима с $\boldsymbol{b}$ и поэтому отлична от $\boldsymbol{a}_{\boldsymbol{\omega}} \boldsymbol{b}$. Следовательно, необходима иная форма итерации.

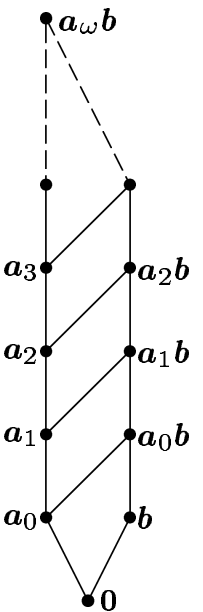

Рис. 1. Структура L-степеней в рассматриваемой модели

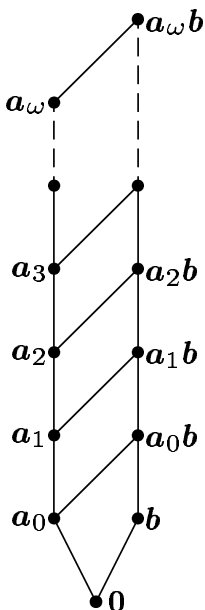

Рис. 2. Структура L-степеней в обычной итерированной модели Сакса с той же системой образующих $\boldsymbol{a}_{n}$ и $\boldsymbol{b}$

(Вещественными) числами мы называем элементы множества $\omega^{\omega}$.

Работа первого автора выполнена при поддержке Калтеха и НИОКР МПС России. Работа второго автора выполнена при поддержке фонда GA ČR, грант № 201/97/0216. 
Tеорема. Пусть $\omega_{1}^{L}$ счетно. Тогда существует генерическое расширение $\mathbf{M}=\mathbf{L}\left[\left\langle a_{n}\right.\right.$ : $n \in \omega\rangle, b]$, порожденное числами $a_{n}$ u $b$, такое, что

i) для любого $n\left\langle\left\langle a_{0}, \ldots, a_{n}\right\rangle, b\right\rangle$ является $\left(\mathrm{Sacks}^{n+1} \times\right.$ Sacks)-генерической над $\mathbf{L}$ последовательностью;

ii) каждое число $x \in \mathbf{M}$ или принадлежит $\mathbf{L}\left[a_{0}, \ldots, a_{n}, b\right]$ для некоторого $n$, или удовлетворяет свойству $\mathbf{L}[x]=\mathbf{L}\left[\left\langle a_{n}: n \in \omega\right\rangle, b\right]$.

Согласно известным свойствам обычных итерированных моделей Сакса (см. [1], [2]) такая модель $\mathbf{M}$ имеет структуру степеней конструктивности чисел, описанную в 1)-4).

1. Форсинг. Следующие рассуждения проводим в модели $\mathbf{L}$.

Пусть $\mathbf{S}=$ Sacks - форсинг Сакса. Для любого $n$ обозначим через $\mathbf{S}^{n}$ итерацию форсинга Сакса длины $n$ с соответствуюшим отношением форсинга $\Vdash_{n}$. Каждое $\tau \in \mathbf{S}^{n}$ есть функция, заданная на множестве $n=\{0, \ldots, n-1\}$, так что $\tau \mid k \Vdash_{k}$ " $\tau(k) \in \breve{\mathbf{S}}$ " для всех $k<n$.

Если $u \in 2^{n}$ и $f$ - функция такая, что каждое ограничение вида $u \uparrow k, k<n$, принадлежит $\operatorname{dom} p$, то через $\left.f\right|_{u}$ обозначим функцию, определенную на $n$ по правилу $(f \mid u)(k)=f(u\lceil k)$ для всех $k<n$.

Определим форсинг $\mathbf{P}$ как семейство всех $p=\left\langle T_{p}, f_{p}\right\rangle$ таких, что

а) $T_{p} \subseteq 2^{<\omega}-$ совершенное дерево;

б) $f_{p}$ - функция, определенная на $T_{p}$ так, что $\left.f_{p}\right|_{u} \in \mathbf{S}^{n}$ для всех $u \in T_{p} \cap 2^{n}$.

Скажем, что $q$ сильнее $p$ (или $q \leqslant p$ ), если $T_{q} \subseteq T_{p}$ и $\left.f_{q}\right|_{u} \leqslant\left. f_{p}\right|_{u}$ в $\mathbf{S}^{n}$ для каждого $u \in T_{q} \cap 2^{n}$. Символом $\Vdash$ обозначим отношение $\mathbf{P}$-форсинга.

Напомним, что $u \in T$ называется расщепляющейся вершиной дерева $T \subseteq 2^{<\omega}$, если вершины $u^{\wedge} 0$ и $u^{\wedge} 1$ принадлежат $T$. Расщепляющаяся вершина уровня $n$ имеет ровно $n$ расщепляющихся вершин ниже себя. Совершенное дерево $T$ содержит в точности $2^{n}$ расщепляющихся вершин на каждом уровне $n$.

Пусть $S$ и $T$ - совершенные деревья. Положим $S \leqslant n$, если $S \subseteq T$ и $n$-е расщепляющиеся уровни в $S$ и $T$ совпадают. Известно, что если $T_{n}-$ совершенные деревья и $T_{0} \geqslant_{0} T_{1} \geqslant_{1} T_{2} \geqslant_{2} \cdots$, то $T=\bigcap T_{n}-$ также совершенное дерево.

Эта конструкция принимает следующую форму для форсинга $\mathbf{P}$.

Пусть $p, q \in \mathbf{P}$. Положим $q \leqslant n p$, если $q \leqslant p, T_{q} \leqslant n T_{p}$ и каждое $u \in T_{q} \cap 2^{m}, m<n$, удовлетворяет свойству $\left.f_{q}\right|_{u} \Vdash_{m}$ " $f_{q}(u) \leqslant n f_{p}(u)$ ". Тогда любая убьвающая цепь $p_{0} \geqslant_{0} p_{1} \geqslant_{1} p_{2} \geqslant_{2} \ldots$ вынуждающих условий $p_{n} \in \mathbf{P}$ имеет нижнюю грань в $\mathbf{P}$.

2. Расширение. Если множество $G \subseteq \mathbf{P}$ является $\mathbf{P}$-генерическим над $\mathbf{L}$, то $T=\bigcap_{p \in G} T_{p}-$ генерическая цепь в $2^{<\omega}$, так что $b=\bigcup T \in 2^{\omega}$ есть число Сакса над $\mathbf{L}$.

Пусть $n \in \omega$. Положим $u=b\left\lceil n, u \in 2^{n}\right.$. Тогда множество $\left.G\right|_{u}=\left\{\left.f_{p}\right|_{u}: p \in G\right\}$ является $\mathbf{S}^{n}$-генерическим над $\mathbf{L}$, т.е. над $\mathbf{L}$ задана $\mathbf{S}^{n}$-генерическая последовательность чисел $\left\langle a_{0}, \ldots, a_{n-1}\right\rangle$, в которой каждое $a_{k} \in 2^{\omega}$ - число Сакса над $\mathbf{L}\left[a_{0}, \ldots, a_{k-1}\right]$. (Разумеется, здесь $a_{k}$ не зависит от выбора $n>k$.) Более того, последовательность $\left\langle\left\langle a_{0}, \ldots, a_{n-1}\right\rangle, b\right\rangle$ является $\left(\mathbf{S}^{n} \times \mathbf{S}\right)$-генерической над $\mathbf{L}$.

Теперь нам осталось доказать утверждение іi) теоремы. Оно вытекает из следующей леммы.

Лемма 1. Предположим, что число $x \in \mathbf{L}[G]$ не принадлежит классу $\mathbf{L}\left[a_{0}, \ldots, a_{n}, b\right]$ ни при каком $n$. Тогда $G \in \mathbf{L}[x]$.

Начнем доказательство с определения. Ростером размера $l$ называется конечная последовательность вида $R=\left\langle u, w_{0}, \ldots, w_{l-1}\right\rangle$, в которой все $u, w_{0}, \ldots, w_{l-1}$ принадлежат $2^{l}$. Ростер $R=$ $\left\langle u, w_{0}, \ldots, w_{l-1}\right\rangle$ можно понимать как условие в $\mathbf{P}$, вынуждающее $\breve{b}$ продолжать $u$, а каждое $\breve{a}_{k}$, $k<l$, продолжать $w_{k}$.

Ростер $R=\left\langle u, w_{0}, \ldots, w_{l-1}\right\rangle$ согласуется $c$ генерическим множеством $G$, если $u \subset b$ и $w_{k} \subset a_{k}$ для всех $k<l$. Ростер $R$ согласуется с условием $p \in \mathbf{P}$, если существует более сильное условие $q \leqslant p$, вынуждающее $R$ быть согласованным с $\breve{G}$. В этом случае имеется наибольшее (т.е. слабейшее) условие $q$ такого вида, обозначаемое как $q=p \nmid R$ (ограничение $p$ на $R$ ): $q$ получается присоединением к $p$ информации о том, что $\breve{b}$ продолжает $u$ и каждое $\breve{a}_{k}, k<l$, продолжает $w_{k}$. 
Будем говорить, что условие $p \in \mathbf{P}$ вполне $n$-расщепляется ниже $l$, если $n$-й уровень расщепления $T_{p}$ целиком ниже $l$ и для любого $u \in T_{p} \cap 2^{m}, m \leqslant n$, имеем

$$
\left.f_{p}\right|_{u} \Vdash_{n} \text { " } n \text {-й уровень расщепления } f_{p}(u) \text { целиком ниже } l \text { ". }
$$

ЛЕмма 2. Предположим, что ростер $R=\left\langle u, w_{0}, \ldots, w_{l-1}\right\rangle$ согласуется с условием $p \in \mathbf{P}$, которое вполне n-расщепляется ниже $l \geqslant n$, а условие $r \in \mathbf{P}$ сильнее чем $p \nmid R$. Тогда существует условие $q \leqslant n p$ такое, что $q \uparrow R$ совпадает $\mathrm{c} r$.

ДоКАЗАТЕльство. Определим $T_{q}$ как множество всех $v \in T_{p}$ таких, что либо $u \nsubseteq v$, либо $v \in T_{r}$. (Тогда каждое $v \in T_{r} \subseteq$-сравнимо с $u$ по выбору $r$.)

Для $v \in T_{q}$ определим $f_{q}(v)$. Положим $f_{q}(v)=f_{r}(v)$, если $u \subseteq v$, и $f_{q}(v)=f_{p}(v)$, если $u, v$ $\subseteq$-несравнимы. Остается рассмотреть случай строгого включения $v \subset u$. Положим $m=\operatorname{dom} v$,

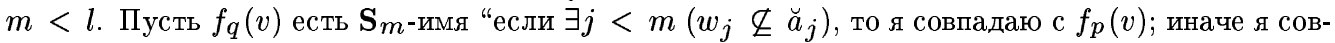
падаю с $\left\{a \in f_{p}(v): w_{m} \subset a \Longrightarrow a \in f_{r}(v)\right\}$ ". Из второй части определения следует, что $q \uparrow R=r$. Пусть $m<n$ и $v \in T_{q} \cap 2^{m}$. Покажем, что $\left.f_{q}\right|_{v} \Vdash_{m}$ " $f_{q}(v) \leqslant n f_{p}(v)$ ". По определению нетривиальным является лишш случай $v=u \nmid m \subset u$. Будем рассуждать в $\mathbf{S}^{m}$-генерическом расширении универсума. Все различия между $f_{p}(v)$ и $f_{q}(v)$ по определению сконцентрированы в области $D=\left\{a \in 2^{\omega}: w_{m} \subset a\right\}$, где $w_{m} \in 2^{l}$. С другой стороны, $n$-й уровень расщепления $f_{p}(v)$ определен нижсе $l$, так что эти различия не нарушают свойства $f_{q}(v) \leqslant n f_{p}(v)$. Следовательно, $q \leqslant n p$.

3. Доказательство леммы 1. Пусть $\breve{x}$ - имя нашего числа $x$. По условию леммы некоторое $p \in G$ вынуждает “ $\breve{x} \notin \mathbf{L}\left[\breve{a}_{0}, \ldots, \breve{a}_{n}, \breve{b}\right]$ ” для любого $n$. Индукцией по $n$ определим

a) последовательность $p=p_{0} \geqslant_{0} p_{1} \geqslant_{1} p_{2} \geqslant_{2} \cdots$ условий $p_{n} \in \mathbf{P}$;

б) последовательность натуральных чисел $l_{0}<l_{1}<l_{2}<\cdots$;

в) функцию $g$, отображающую ростеры размера $l_{n}$ в $\Sigma \cup\{\perp\}$, где $\Sigma$ - множество всех функций $\sigma$ таких, что $\operatorname{dom} \sigma \subseteq \omega$ конечно и $\operatorname{ran} \sigma \subseteq\{0,1\}$, a $\perp$ - формальный символ для отделения несущественных вариантов

так, что для каждого $n$ и любого ростера $R$ размера $l_{n}$

$$
p_{n+1} \Vdash \text { " } R \text { согласуется с } \breve{G} \Longleftrightarrow g(R) \neq \perp \text { и } g(R) \subset \breve{x} \text { ". }
$$

Тогда любая нижняя грань $q \in \mathbf{P}$ последовательности условий $p_{n}$ вынуждает $\breve{G}$ быть единственньм генерическим множеством, согласованным со всеми ростерами $R$, удовлетворяющими свойству $g(R) \subset \breve{x}$. Следовательно, $q$ вынуждает " $\breve{G} \in \mathbf{L}[\breve{x}]$ ”, что и требовалось.

Допустим, что $p_{n}$ уже определено. Покажем, как определить $l_{n}$, действие $g$ на ростеры размеpa $l_{n}$ и условие $p_{n+1}$.

Первая часть. Согласно известным свойствам форсинга Сакса и его конечных итераций существуют условие $q \leqslant n p_{n}$ и натуральное число $l_{n}>l_{n-1}$ такие, что $T_{q}=T_{p_{n}}$ и $q$ вполне $n$-расщепляется ниже $l_{n}$. Зафиксируем перечисление $\left\langle R_{k}^{0}, R_{k}^{1}\right\rangle, k<K$, всех пар различных ростеров размера $l_{n}$. Индукцией по $k \leqslant K$ определим

а) условия $q=q_{0} \geqslant_{n} q_{1} \geqslant n q_{2} \geqslant n \ldots \geqslant n q_{K}$ в $\mathbf{P}$;

б) натуральные числа $m_{k} \in \omega$ и $i_{k} \in\{0,1\}$

так, что для каждого $k<K$ выполнено

$$
q_{k+1} \Vdash\left\{\begin{array}{l}
\text { "если } R_{k}^{0} \text { согласуется с } \breve{G}, \text { то } \breve{x}\left(m_{k}\right)=i_{k} ", \\
\text { "если } R_{k}^{1} \text { согласуется с } \breve{G}, \text { то } \breve{x}\left(m_{k}\right) \neq i_{k} \text { ”. }
\end{array}\right.
$$

Затем положим $p_{n+1}=q_{K}$ и для любого ростера $R$ размера $l_{n}$

$$
g(R)= \begin{cases}\left\{\left\langle m_{k}, i_{k}\right\rangle: k<K, R=R_{k}^{0}\right\}, & \text { если } R \text { согласуется с } q_{K}, \\ \perp & \text { в противном случае. }\end{cases}
$$

Такой выбор, очевидно, влечет (1). 
Вторая часть. Теперь, имея $q_{k}$, определим $q_{k+1}, m_{k}$ и $i_{k}$. Построение включает два шага.

Шаг 1. Находим пару промежуточных условий $q^{0}$ и $q^{1}$. Если ростер $R_{k}^{0}$ не согласуется с $q_{k}$, то положим $q^{0}=q^{1}=q_{k}$ и перейдем к шагу 2 . Допустим, что $R_{k}^{0}$ coгласуется $c q_{k}$. Напомним, что $R_{k}^{0}$ - pостер размера $l_{n}$, т.е. $R_{k}^{0}=\left\langle u, w_{0}, \ldots, w_{l_{n}-1}\right\rangle$, где $u$ и $w_{j}$ принадлежат $2^{l_{n}}$.

Поскольку $q_{k}$ вынуждает “ $\breve{x} \notin \mathbf{L}\left[\breve{a}_{0}, \ldots, \breve{a}_{l_{n}}, \breve{b}\right]$ ”, найдутся условия $r^{0}, r^{1} \in \mathbf{P}$, которые сильнее $q_{k} \uparrow R_{k}^{0}$ и удовлетворяют свойствам $T_{r 0}=T_{r 1}$ (заметим, что тогда $u \in T_{r_{0}}=T_{r_{1}}$ ) и $\left.f_{r^{0}}\right|_{u}=\left.f_{r^{1}}\right|_{u}$, а также найдется число $m_{k} \in \omega$ такое, что $r^{0} \Vdash$ “ $\breve{x}\left(m_{k}\right)=0$ ” и $r^{1} \Vdash$ “ $\breve{x}\left(m_{k}\right)=1$ ".

Существование условий $q^{0}$ и $q^{1}$ в $\mathbf{P}$, для которых $q^{i} \leqslant n q_{k}$ и $q^{i} \uparrow R_{k}^{0}=r^{i}, i=0,1$, обеспечивается леммой 2 . Более того, как показывает анализ доказательства леммы 2 , если условия $r^{i}$ выбраны, то условия $q^{i}$ можно выбрать так, чтобы $T_{q^{0}}=T_{q^{1}}$ и $f_{q^{0}}(v)=f_{q^{1}}(v)$ для всех $v \in T_{q^{0}}=T_{q^{1}}$, не удовлетворяющих включению $u \subseteq v$. В частности, выполнено $q^{0} \uparrow R=q^{1} \uparrow R$ для любого ростера размера $l_{n}$.

Шаз 2. Если $R_{k}^{1}$ не согласуется с $q^{0}$ (значит, и с $q^{1}$ согласно сказанному выше), то положим $q_{k+1}=q^{0}$ и $i_{k}=0$. Допустим, что $R_{k}^{1}$ coгласуетсл $c r$.

Условие $r \leqslant q^{0} \uparrow R_{k}^{1}$ определяет значение $\breve{x}\left(m_{k}\right)$ равным, скажем, 0 . Положим $i_{k}=1$. Согласно доказанному выше $r \leqslant q^{1} \uparrow R_{k}^{1}$. С помошью леммы 2 получаем условие $q \leqslant n q^{1}$ такое, что $q \uparrow R_{k}^{1}=r$. Итак, $q \leqslant n q_{k}, q \uparrow R_{k}^{1}=r \Vdash$ “ $\breve{x}\left(m_{k}\right)=0$ ”, и если $q$ согласуется с $R_{k}^{0}$, то $q \uparrow R_{k}^{0} \leqslant q^{1} \uparrow R_{k}^{0}$. Следовательно, $q \uparrow R_{k}^{0} \Vdash$ “ $\breve{x}\left(m_{k}\right)=i_{k}=1$ ”.

Таким образом, условие $q_{k+1}=q$ имеет требуемые свойства.

\section{СПИСОК ЦИТИРОВАННОЙ ЛИТЕРАТУРЫ}

1. Baumgartner J. E., Laver R. // Ann. Math. Logic. 1979. V. 17. P. 271-288. 2. Kanovei V. On non-wellfounded iterations of perfect set forcing // J. Symbolic Logic (to appear). 